\title{
Chitosan-lignosulfonates sono-chemically prepared nanoparticles: Characterisation and potential applications
}

\author{
Suyeon Kim ${ }^{\mathrm{a}, 1,3}$, Margarida M. Fernandes ${ }^{\mathrm{a}, 2,3}$, Teresa Matamáa, ${ }^{\mathrm{a}, \mathrm{b}}$, Ana Loureiro ${ }^{\mathrm{a}, \mathrm{b}}$, Andreia C. Gomes ${ }^{\mathrm{b}, *}$, \\ Artur Cavaco-Paulo ${ }^{\mathrm{a}}$ \\ a Textile Engineering Department, University of Minho, Campus Azurém, 4800-058 Guimarães, Portugal \\ ${ }^{\mathrm{b}}$ CBMA - Centre of Molecular and Environmental Biology, Department of Biology, University of Minho, Campus Gualtar, 4710-057 Braga, Portugal
}

\section{A R T I C L E I N F O}

\section{Article history:}

Received 16 July 2012

Received in revised form 17 October 2012

Accepted 22 October 2012

Available online $\mathrm{xxx}$

\section{Keywords:}

Chitosan

Lignosulfonates

Nanoparticles

Ultrasound

Antimicrobial activity

\begin{abstract}
A B S T R A C T
Due to their recognised properties of biocompatibility, biodegradability and sustainability, chitosan nanocarriers have been successfully used as new delivery systems. In this work, nanoparticles combining chitosan and lignosulfonates were developed for the first time for cosmetic and biomedical applications. The ability of lignosulfonates to act as a counter polyion for stabilisation of chitosan particles, generated using high intensity ultrasound, was investigated. Several conditions for particles preparation were tested and optimised and the resulting nanoparticles were comprehensively characterised by measuring particle size, zeta potential and polydispersity index. The $\mathrm{pH}$ of chitosan solution, sonication time and the presence of an adequate surfactant, poloxamer 407, were determinant factors on the development of smaller particles with low polydispersity index (an average particle size of $230 \mathrm{~nm}$ was obtained at pH 5 after 8 min of sonication). The beneficial effects of lignosulfonates complex on chitosan nanoparticles were further characterised. Greater stability to lysozyme degradation, biocompatibility with human cells and antimicrobial activity was found upon lignosulfonates incorporation into chitosan nanoparticles. Furthermore, these particles were able to incorporate a hydrophilic model protein - RNase A. A burst release was observed when nanoparticles were loaded with low amount of protein while with high protein content, a sustained release was found, suggesting that the protein cargo maybe loaded both at the surface as in the bulk of the particle, depending on the concentration of drug incorporated.
\end{abstract}

(c) 2012 Elsevier B.V. All rights reserved.

\section{Introduction}

Biodegradable nanoparticles have attracted great attention due to their capacity not only to protect proteins and peptides from degradation but also to desirable release profiles [1]. The development seen in the last years in nanotechnology and delivery systems has been achieved mainly through new formulations with improved stability and drug encapsulation efficiency, optimised particle size and specific targeting [2].

Nanoparticles are very important vehicles due to their unique features such as their surface to mass ratio, quantum properties, and potential for absorbing and carrying other molecules [3]. Therefore the search for the appropriate carriers is

\footnotetext{
* Corresponding author. Tel.: +351253 601511; fax: +351253678980. E-mail address: agomes@bio.uminho.pt (A.C. Gomes).

1 Present address: Interdisciplinary Research Laboratory, Pontificia Universidad Católica del Perú (PUCP), Av. Universitaria 1802, San Miguel Lima 32, Peru.

2 Present address: Group of Molecular and Industrial Biotechnology, Department of Chemical Engineering, Universitat Polìtecnica de Catalunya, Ernest Lluch s/n, 08222 Terrassa, Spain.

${ }^{3}$ Both authors equally contributed to this work.
}

growing. They must meet several requirements like the ability to incorporate and release the compounds of interest, increased formulation stability and biocompatibility. In addition, the possible adverse effect of residual materials after delivery should be negligible [3]. Chitosan is composed of 2-amino-2-dedoxyD-glucose and 2-acetamino-dedoxy-D-glucose units linked with $\beta-(1 \rightarrow 4)$ bonds and it is derived from alkaline deacetylation of chitin, one of the most abundant natural polysaccharides [1]. The properties of chitosan depend mostly on its molecular weight and degree of deacetylation [4]. The availability of free amino groups confers to chitosan a net positive charge favouring ionic interaction with many negatively charged polymers or surfaces. Chitosan has great potential for pharmaceutical applications due to its biocompatibility, biodegradability, antibacterial activity, high charge density and mucoadhesion. The mucoadhesive properties arise from the molecular attractive forces due to the electrostatic interaction between positively charged chitosan and negatively charged mucosal surfaces [5,6]. Recently, chitosan based delivery systems have been used for the improved delivery and controlled release of peptides, proteins, oligonucleotides and plasmids [4,7]. It was demonstrated that they protect sensitive bioreactive macromolecules from enzymatic 
degradation in vivo and chemical degradation during storage [8].

Various methods have been developed for the production of chitosan nanoparticles such as ionotropic gelation, spray drying, emulsification and coacervation [5]. However, the addition of an anionic compound, like alginate, tripolyphosphate (TPP), carrageenan or polyelectrolyte, is the most common technique for the preparation of chitosan nanoparticles. The development of stable chitosan microsphere is limited by the ionic interaction between chitosan and the anionic compound. The search for new non-toxic and functional anionic compounds is therefore of great interest.

In this work, sulfonated lignin was used for the first time as a counter ion polymer to produce chitosan nanoparticles with increased stability. Lignin is also a biodegradable polymer and its physical and chemical properties can be modulated depending on the extraction technology [9]. In water, sulfonated lignin acts as an anionic polyelectrolyte due to sulfonate groups $\left(\mathrm{SO}_{3}{ }^{-}\right)$. Lignosulfonates have high molecular weight and contain functional sulfonate groups which make them water soluble anionic polymers. The water soluble lignosulfonates have many distinct applications. The major use of lignosulfonates is as dispersion agents. Ligninbased products are also used in ceramics, in textile dyeing as dispersants, in pesticides, and as binders in briquetting and animal feed [10-12]. Moreover, the antioxidant and antimicrobial activities have extended their potential applications $[11,13]$.

In this work, chitosan-lignosulfonate nanoparticles, ionically crosslinked, were produced using ultrasonication. Ultrasonication has been broadly used for the preparation and processing of polymer nanoparticles. This tool is particularly effective in reducing particle size and narrowing size distributions [14]. As it was the first attempt to incorporate lignosulfonates in chitosan particles, a strong emphasis was given to the properties of lignosulfonates containing particles. For this purpose, the particles optimised in terms of size and polydispersity were further characterised regarding degradation, loading efficiency, releasing properties, as well as cytotoxicity. In addition, the antimicrobial activity was also evaluated using two types of Gram-negative bacteria and two types of Gram-positive bacteria.

\section{Experimental}

\subsection{Materials}

Chitosan from shrimp shells (deacetylation degree $\geq 75 \%$; viscosity $200 \mathrm{cps}$ ) was purchased from Sigma-Aldrich (St. Louis, USA). The sulfonated lignin compounds isolated from hardwood (DP401: calcium lignosulfonates) were kindly supplied by Borregaard-LignoTech (Sarpsborg, Norway). Commercial ediblegrade vegetable oil was used as organic phase. Non-ionic surfactants, poloxamer 407 and Tween 80, Lysozyme and RNase A and all other chemicals were purchased from Sigma-Aldrich.

\subsection{Chitosan-lignosulfonates (CS-LS) particles preparation}

CS-LS particles were sonochemically produced by an adaptation of Suslick method [15]. The formation of particles was processed by incorporation of CS and LS solutions, $30 \mathrm{~mL}$ and $15 \mathrm{~mL}$, respectively, in the presence of $5 \mathrm{~mL}$ of organic phase (vegetal oil). The highintensity ultrasonic probe was positioned at the aqueous-organic interface and several reaction factors were considered and evaluated.

\subsubsection{Effect of $\mathrm{pH}$ of chitosan (CS) and lignosulfonates (LS) solutions}

Solutions containing $2 \mathrm{~g} \mathrm{~L}^{-1}$ of chitosan were prepared in $1 \%$ acetic acid, then the $\mathrm{pH}$ was adjusted to $4.5,5$ and 6 using $1 \mathrm{M}$ of $\mathrm{NaOH}$. After $\mathrm{pH}$ adjustment, solutions were cleared using filter paper. The LS solutions $\left(1 \mathrm{~g} \mathrm{~L}^{-1}\right)$ were prepared in distilled water without $\mathrm{pH}$ adjustment $(\mathrm{pH} \sim 8)$ and in PBS buffer $(\mathrm{pH} 7.4)$.

\subsubsection{Effect of ultrasonication time}

Ultrasonic treatments were carried out using an ultrasound probe of $13 \mathrm{~mm}$ in diameter with a titanium micro-tip that was immersed to the borderline between sample solution and oil phase (20 kHz Sonics, $40 \%$ amplitude). Ultrasonication was carried out at fixed amplitude with durations of 2, 5, 8 and $10 \mathrm{~min}$. During ultrasonication, the samples were maintained in ice and then samples were kept at $4{ }^{\circ} \mathrm{C}$ for $24 \mathrm{~h}$ to complete the phases separation before being analysed.

\subsubsection{Effect of stabilising agents and their concentrations}

Non-ionic surfactants, poloxamer 407 and Tween 80, were introduced in the process of CS solution preparation. Surfactant concentrations of $0.05,0.1,0.2,0.3,0.4,0.5,1$ and $1.5 \%(\mathrm{v} / \mathrm{v})$ were applied to $2 \mathrm{~g} \mathrm{~L}^{-1} \mathrm{CS}$ solutions. The particles were produced after the addition of $\mathrm{LS}\left(1 \mathrm{~g} \mathrm{~L}^{-1}\right)$ by ultrasonication for $8 \mathrm{~min}$.

\subsubsection{Effect of concentrations of chitosan (CS) and lignosulfonates (LS)}

Several concentrations of CS and LS were used in the formulation and their effects on particle diameters were studied. CS-LS particles were produced in the presence of poloxamer 407 surfactant ( $1 \%$, $\mathrm{v} / \mathrm{v}$ ) by ultrasonication for $8 \mathrm{~min}$.

\subsection{Biostability of CS and CS-LS nanoparticles}

In vitro biodegradation tests of CS nanoparticles with and without LS were monitored in the absence and presence of lysosyme, a glycoside hydrolase enzyme, by incubating $1 \mathrm{~mL}$ of particles emulsion with $5 \mathrm{~mL}$ PBS buffer, pH 7.4 in the presence or absence of $0.5 \mathrm{~g} \mathrm{~L}^{-1}$ lysozyme at $37^{\circ} \mathrm{C}$ for $1 \mathrm{~h}, 3$ days and 8 days. Samples of $0.25 \mathrm{~mL}$ were taken and filtered with a $0.2 \mu \mathrm{m}$ filter in order to remove intact particles from the hydrolysis products. The degraded chitosan was determined in the filtrate using the ninhydrin test. The degradation of nanoparticles was quantitatively evaluated as the amount of hydrolysed chitosan leached from the nanoparticles, previously using chitosan as a standard for the calibration curve.

\subsection{Evaluation of protein loading capacity $(L C)$ and protein incorporation}

The RNase A loaded CS-LS nanoparticles were prepared using different quantities of protein (final concentration of RNase in solutions: $0.2,0.5,1.0$ and $2.0 \mathrm{mg} \mathrm{mL}^{-1}$ ). The protein was solubilised in the $1 \mathrm{~g} \mathrm{~L}^{-1}$ LS solution prior to sonication. The protein incorporation was determined through the quantification of free, non-encapsulated RNase A. The separation of the free protein from nanoparticles emulsion was accomplished by centrifugation using Amicon ultra centrifugal filter (Ultra-15 MWCO $100 \mathrm{kDa}$ ). The protein quantification on aqueous medium was made via the Lowry method [16], using BSA as standard and using Sigma test kit $\mathrm{n}^{\circ} \mathrm{P}$ 5656. The LC was obtained following Eq. (1) [17].

$$
\text { loading capacity }(\%)=\frac{\text { total RNase A weight }- \text { free RNase A weight }}{\text { nanoparticles particles weight }} \times 100
$$

\subsection{In vitro release behaviour}

CS-LS nanoparticles loaded with different concentrations of RNase A were incubated in PBS and in the absence and presence of lysosyme at $37^{\circ} \mathrm{C}$ for different periods of time. After $30 \mathrm{~min}, 1$, $2,3,4,24$ and $48 \mathrm{~h}$ the suspensions were centrifuged using Amicon units. The amount of released protein was quantified by the 
Lowry method. Protein unloaded CS-LS nanoparticles, under the same conditions, were used as controls in order to correct for the possible interference of CS or LS.

\subsection{Physicochemical characterisation of nanoparticles}

The determination of particle size and zeta potential was carried out using a Zetasizer Nano Series (Malvern Instruments Inc., Worcester, UK) after appropriate dilution of nanoparticles using ultrapure-grade water.

\subsection{Antimicrobial activity test}

The antibacterial effect of LS, CS and CS-LS particles as their ability to inhibit bacterial growth was evaluated using a turbidimetric method. A Gram-negative bacteria, Escherichia coli HB101, and two Gram-positive bacteria, Staphylococcus aureus ATCC6538 and Bacillus subtilis 48886 were tested. The microorganisms were grown in Muller Hinton ( $\mathrm{MH}$ ) broth (Fluka Analytical, Buchs, Switzerland) incubated at $37^{\circ} \mathrm{C}$. All the inocula were grown overnight and diluted in $\mathrm{MH}$ broth till an optical absorbance of 0.01 at $600 \mathrm{~nm}$. Incubations were carried out in a 96-wells plate at $200 \mathrm{rpm}$ for at least $390 \mathrm{~min}$. The assay was initiated by mixing $135 \mu \mathrm{L}$ of the diluted culture or just sterile MH broth (blanks) and $15 \mu \mathrm{L}$ of the nanoparticle suspensions. The CS, LS and CS-LS nanoparticles suspensions were thoroughly washed with sterile PBS. Two controls were performed, one with $50 \mu \mathrm{g} \mathrm{mL}^{-1}$ ampicilin and the other with $0.1 \times$ phosphate buffered saline (no treatment), the buffer used to dilute the nanoparticles. The bacterial growth was monitored by the optical density at $600 \mathrm{~nm}$ over time corrected for the background absorbance of each blank, using a SepctraMax 340 PC spectrophotometer (Molecular devices, Sunnyvale, CA, USA). The specific growth rate $\left(\mathrm{min}^{-1}\right)$ was obtained from the exponential phase time points for each growth curve and related to the specific growth rate $\left(\mathrm{min}^{-1}\right)$ of the control (PBS buffer). The error bars for each data point were the standard deviation of three parallel measurements.

\subsection{Cytotoxicity evaluation of nanoparticles}

For the cytotoxicity evaluation, only the CS, LS and CS-LS nanoparticles were further sterilised with $0.45 \mu \mathrm{m}$ filters in order to avoid cell culture microbial contamination. Different dilutions of these suspensions were prepared in complete culture medium. The culture medium itself was used as a negative control, whereas a $30 \%$ dimethyl sulfoxide (DMSO) solution (Sigma) prepared in culture medium was used as a toxic positive control.

\subsubsection{Culture of human skin fibroblasts cell line (BJ-5ta)}

The BJ-5ta cell line (normal human skin fibroblasts) was maintained according to ATCC recommendations (4 parts Dulbecco's Modified Eagle's Medium (DMEM) containing $4 \mathrm{mM}$ L-glutamine, $4.5 \mathrm{~g} \mathrm{~L}^{-1}$ glucose, $1.5 \mathrm{gL}^{-1}$ sodium bicarbonate, and 1 part of Medium 199, supplemented with $10 \%(\mathrm{v} / \mathrm{v}$ ) of foetal bovine serum (FBS), $1 \%(\mathrm{v} / \mathrm{v}$ ) of penicillin/streptomycin solution and $10 \mu \mathrm{g} \mathrm{mL}-1$ Hygromycin B). The cells were maintained at $37^{\circ} \mathrm{C}$ in a humidified atmosphere with $5 \% \mathrm{CO}_{2}$. Culture medium was replaced every 2 days.

\subsubsection{AlamarBlue assay}

Cells were seeded at a density of 10,000 cells/well on a 96-well tissue culture polystyrene (TCPS) plates (TPP, Switzerland) the day before experiments and then they were exposed to the particles and incubated at $37^{\circ} \mathrm{C}$ in a humidified atmosphere with $5 \% \mathrm{CO}_{2}$. Cells were examined at 24,48 and $72 \mathrm{~h}$ for signs of toxicity, using AlamarBlue assay (AlamarBlue ${ }^{\circledR}$ Cell Viability Reagent, Invitrogen).
Resazurin, the active ingredient of AlamarBlue ${ }^{\circledR}$ reagent, is a nontoxic, cell permeable compound that is blue in colour and reduced to resorufin, red colour compound, by viable cells. AlamarBlue ${ }^{\circledR}$ reagent was diluted in culture medium according to the manufacturer instructions and it was added to each well after aspirating the culture medium containing the particles. After $4 \mathrm{~h}$ of incubation at $37^{\circ} \mathrm{C}$ the absorbance at $570 \mathrm{~nm}$ was measured, using $600 \mathrm{~nm}$ as a reference wavelength, in a microplate reader (Spectramax 340PC). The quantity of resorufin formed is directly proportional to the number of viable cells. The error bars for each data point were the standard deviation of three parallel measurements

\section{Results and discussion}

\subsection{Preparation and characterisation of chitosan-lignosulfonates (CS-LS) particles}

In this study, the high intensity of ultrasound radiation was applied as an inexpensive method to produce nanoparticles comprising CS and LS. The sulfonic-based lignin compound was used for the first time as the counter ion polymer to react with the positively charged CS polymer. CS has a $\mathrm{p} K_{\mathrm{a}}$ of approximately 6.5 on the amino groups and at $\mathrm{pH}$ lower than 6.5 , amines are protonated acquiring therefore a polycationic behaviour [4]. In aqueous reaction medium, CS and LS strongly bond by electrostatic interactions. The positive charge on CS gives rise to strong electronic interaction with negatively charged surfaces or polymers. This allows for the high bioavailability and biocompatibility that characterise CS. Furthermore, the charge property, particle size, density, viscosity, deacetylation degree and molecular weight are also important factors which influence the properties and applicability of pharmaceutical formulations based on CS [5].

A detailed characterisation of CS-LS particles was performed. Fig. 1A shows that each type of polymer composition present distinct particle properties. The zeta-potential values, in particular, demonstrate well the electronic charge character of the particles that is inherent to the functional groups of the polymer. The combination of both LS and CS resulted in positively charged particles (CS-LS: $+30.85 \mathrm{mV}$ ) which may be explained by an unequal physical distribution of CS and LS within the particle, with CS being located predominantly on the outer layer. The hypothetical structure of particles produced by ionic interaction between CS and LS is schematically presented in Fig. 1B.

The physical stability and in vivo distribution of nanoparticles are affected by their mean size, polydispersity and surface charge [14]. Aiming at particle smaller sizes and narrower size distributions, the influence of various reaction parameters were studied, in particular, the $\mathrm{pH}$ of CS solutions and the duration of ultrasonication.

The size of particles is very important for an efficient applicability. When particle size is smaller, nanoparticles have larger surface to volume ratio with a more controlled release of insoluble drugs [5]. The pH of CS was adjusted to 4.5, 5 and 6 using $1 \mathrm{M}$ of $\mathrm{NaOH}$ and several sonication periods were tested. By observing Fig. 2, both factors seem to be determinant factors in the preparations of CS-LS particles. Tang et al. have reported that the ultrasonication led to a decrease in mean diameter and polydispersity of particle size when the duration time or amplitude was increased [14]. According to the results (Fig. 2), a concomitant decrease of particle size and polydispersity is also observed with increasing sonication time. Eight minutes are sufficient to produce smaller and less polydisperse nanoparticles. This was the time chosen to produce the CS-LS particles for further testing. A range of $\mathrm{pH}$ values of CS solutions were also studied in parallel. The $\mathrm{pH}$ is determinant in the formation of CS and LS complexes, since it establishes the CS protonation degree 
A
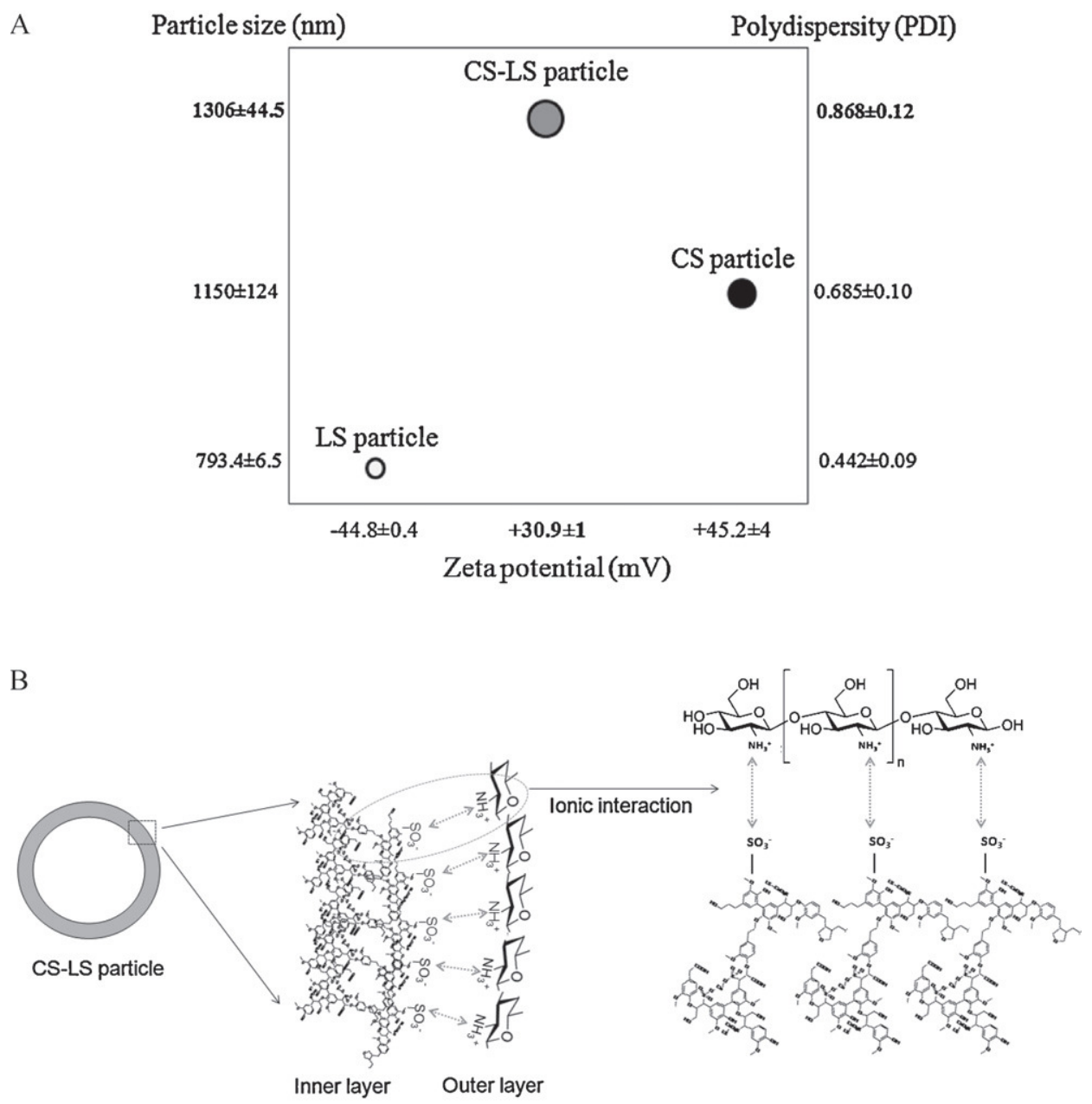

Fig. 1. (A) Average diameter, polydispersity and zeta potential of CS, LS and CS-LS particles, produced by ultrasonication for 8 min before further optimisation. (B) Scheme of CS-LS particles formation based on ionic interactions between $\mathrm{NH}_{3}{ }^{+}$groups of $\mathrm{CS}$ and $\mathrm{SO}_{3}{ }^{-}$groups of LS.

and therefore the ability of CS to interact with the counter ions and its self-association [7]. When the $\mathrm{pH}$ of CS solution is not adjusted ( $\mathrm{pH}$ values around 3.5), the sizes of particles are highly dispersed (Fig. 2B). Adjusting the $\mathrm{pH}$ of $\mathrm{CS}$ to 5 was the best condition for CS-LS particle formation for both smaller particles' diameter and lower polydispersity. Duration of sonication does not influence particle charge (Fig. 2C). The zeta potential is, however, highly affected by the $\mathrm{pH}$ of CS solutions used for particle production. As expected, the surface charge of particles negatively correlated with $\mathrm{pH}$ values of initial solutions (Fig. 2C). The use of PBS buffer to solubilise LS affected the particle size distribution but not the average particles' diameter (Fig. 2A and B). A much lower zeta potential was obtained when PBS was used as solvent comparing to distilled water for the preparation of LS (Fig. 2C).

After achieving the optimum conditions for CS-LS particles production, the effect of a stabiliser agent and the concentration of CS and LS were studied. Non-ionic surfactants (poloxamer 407 and Tween 80) which are commonly used for food, medical and cosmetic applications were tested. They exhibited different effects on particles' size. With poloxamer, the diameter decreased sharply with the increase of surfactant amount in the reaction medium till $0.2 \%$ (v/v) (Fig. 3A). From 0.2 to $1 \%$ added poloxamer, the reduction in particle size was less prominent, while size increased in presence of more than $1 \%$ poloxamer. The polydispersity also reaches a minimum at $1 \%(\mathrm{v} / \mathrm{v})$ of poloxamer concentration. The addition of another surfactant, Tween 80 , did not produce coherent results and the particle size as well as the polydispersity values were too high when compared with those obtained using poloxamer (Fig. 3B). This result shows that poloxamer was more efficient than Tween 80 in decreasing CS-LS particle size and polydispersity.

After optimising the $\mathrm{pH}$, sonication time and surfactant for particles production, the effects of CS and LS concentrations were also studied. The concentration of chitosan did not affect significantly the average particle diameter. At $10 \mathrm{gL}^{-1}$ of chitosan solution, though the smallest size and the lowest polydispersity of CS-LS nanoparticles (size: $221 \mathrm{~nm}$, polydispersity: 0.15) were obtained, the high viscosity was a major drawback in the mixing procedure. Considering the results, to test the influence of LS concentration, $2 \mathrm{~g} \mathrm{~L}^{-1}$ chitosan was the chosen CS concentration (size: $226 \mathrm{~nm}$, polydispersity: 0.21 ). The best chitosan/lignosulfonates ratio found was $2: 1$ corroborating previous results made obtained this ratio (Fig. 4A and B).

Nanoparticles' chemical composition, size, shape, surface charge, hydrophobicity [18] and the presence or absence of functional groups or other chemicals [19] are important characteristics that will define their potential application. Nanoparticles are widely applied on medical and cosmetic areas for drug delivery purposes. In medical application, if orally and intravenously administrated, the optimal size of nanoparticles should range from 10 to $200 \mathrm{~nm}$. The micrometre size below 200 allows for systematic administration in circulation system and also into targeted tissue as well as enhance the ability of nanoparticles to evade the biological particulate filters, such as the reticuloendothelial system [20]. On the other hand, for cosmetic applications, e.g. topically applied 
(A)

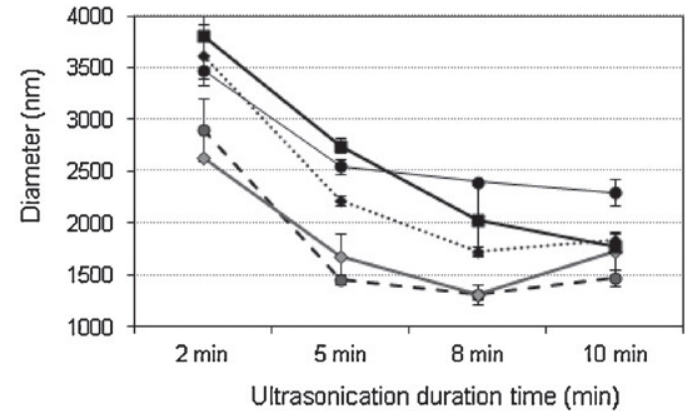

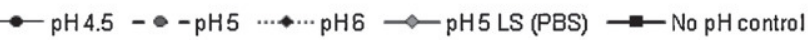

(B)

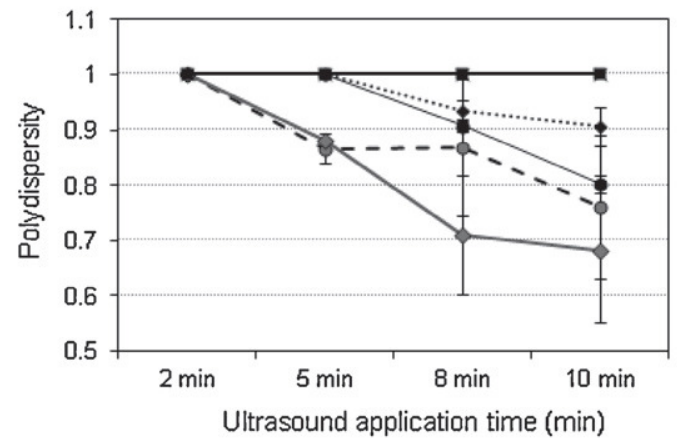

$\bullet \mathrm{pH}_{4} .5-\bullet-\mathrm{pH} 5 \cdots \leftrightarrow \cdots \mathrm{pH} 6 \multimap \mathrm{pH} 5$ LS(PBS) $\rightarrow$ No pH control

(C)

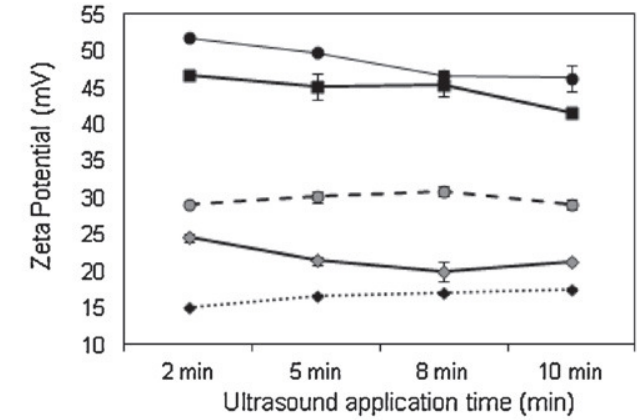

$\rightarrow-$ No pH control $\bullet-\mathrm{pH} 4.5-\bullet-\mathrm{pH}_{5} \cdots \cdots \mathrm{pH} 6 \multimap \mathrm{pH} 5$ LS(PBS)

Fig. 2. Influence of solutions $\mathrm{pH}$ and ultrasonication time on the mean particles diameter (A), polydispersity (B) and of zeta-potential (C) of CS-LS particles. The $\mathrm{pH}$ values that appear in the legend are the $\mathrm{pH}$ for the $\mathrm{CS}$ solutions; no $\mathrm{pH}$ control means that the $\mathrm{pH}$ of CS solution was not adjusted. The LS solution was prepared in water except for the assay pH 5 LS (PBS), this curve was obtained when the LS solution was prepared in PBS pH 7.4.

on skin, sizes above $200 \mathrm{~nm}$ can be successfully used. Despite the fact that evidences have been showing that nanoparticles $>10 \mathrm{~nm}$ in diameter are unlikely to penetrate through the stratum corneum into viable human skin, they are able to accumulate in the hair follicle openings, especially after massage [21].

After the optimisation process, the CS-LS particles were characterised with an average particle size of $230 \mathrm{~nm}$, which constitute a suitable carrier for being applied topically on the skin, e.g. for cosmetic applications.

\subsection{CS-LS nanoparticles biostability}

The degradation profile of CS-LS nanoparticles was tested in the presence of either PBS or lysozyme, a mammalian enzyme present in healthy human secretions $[22,23]$ that is capable of degrading chitosan [7]. Fig. 5A shows the chitosan degradation profile of CS
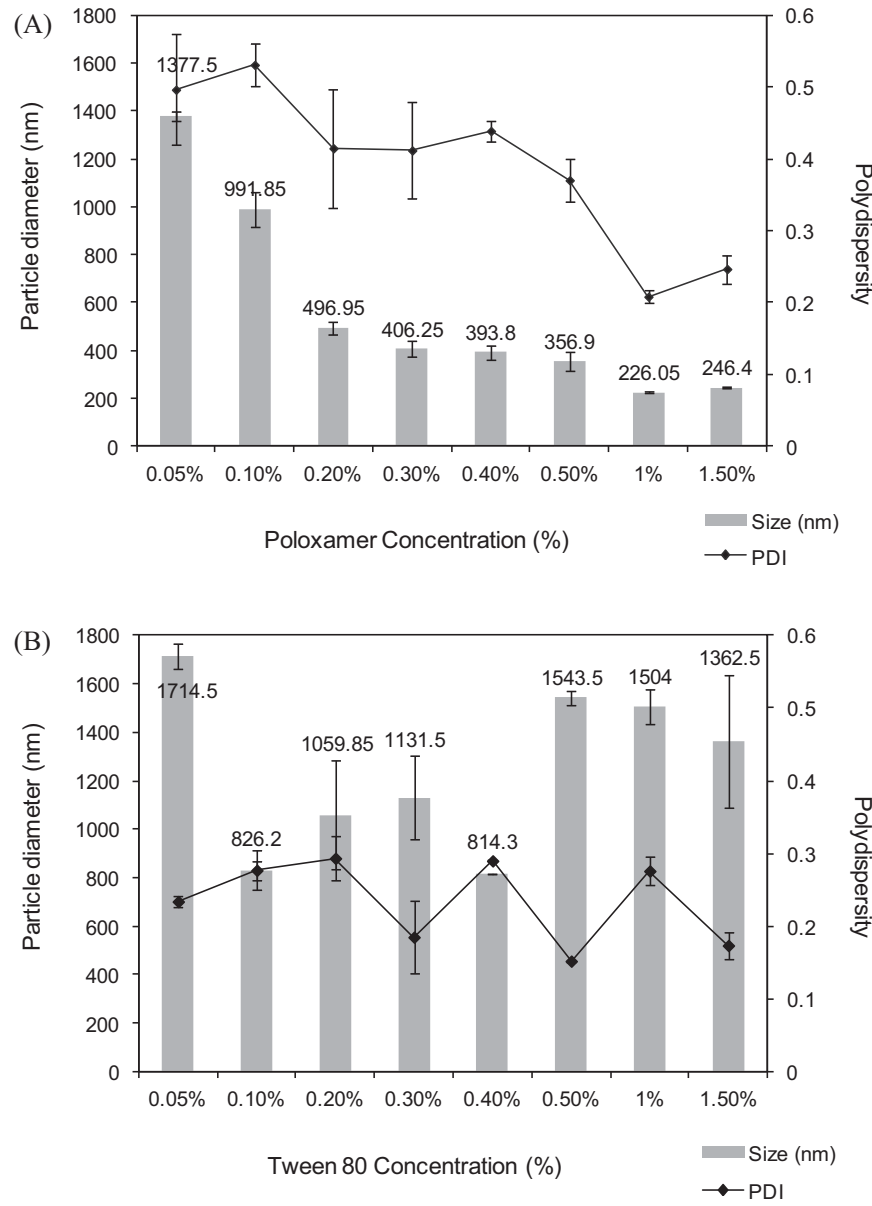

Fig. 3. Mean particles diameter and polydispersity of CS-LS nanoparticles as a function of concentration of poloxamer 407 (A) and Tween 80 (B) surfactant.

and CS-LS nanoparticles after $1 \mathrm{~h}, 3$ days and 8 days of incubation. The nanoparticles were considered stable after $1 \mathrm{~h}$ and 3 days of incubation in either PBS or lysozyme (Fig. 5A). However, the stability of CS nanoparticles was clearly diminished after 8 days of incubation, in particular in the presence of lysozyme. CS-LS particles were even more susceptible to lysozyme activity for the same period. It has been reported that the stability of CS complexes with anionic polyelectrolyte depends mainly on the degree of ionisation of each of the oppositely charged polyelectrolytes [24]. Complexes are stabilised by different types of intermolecular bonds, ionic, covalent, van der Walls interactions and hydrogen bonding [25]. The type and strength of the bonds determine the different degradation profiles.

The incorporation of lignosulfonates in CS particles seems to favour the degradation of chitosan in the presence of lysozyme and it does not affect the stability in PBS. These results indicate that these nanoparticles are potential candidates for drug delivery carriers since they are stable under physiological conditions, and they can be further tuned for drug release.

\subsection{Entrapment and release behaviour of RNase A in CS-LS nanoparticles}

Chitosan has been extensively investigated for the development of novel drug delivery nanosystems. There are many factors affecting the entrapment efficiency of drugs or proteins into nanoparticles. The chemical nature of the drug, the ratio of drug to chitosan in the formulation and the stirring speed (mechanical energy applied) are some examples. These parameters also 

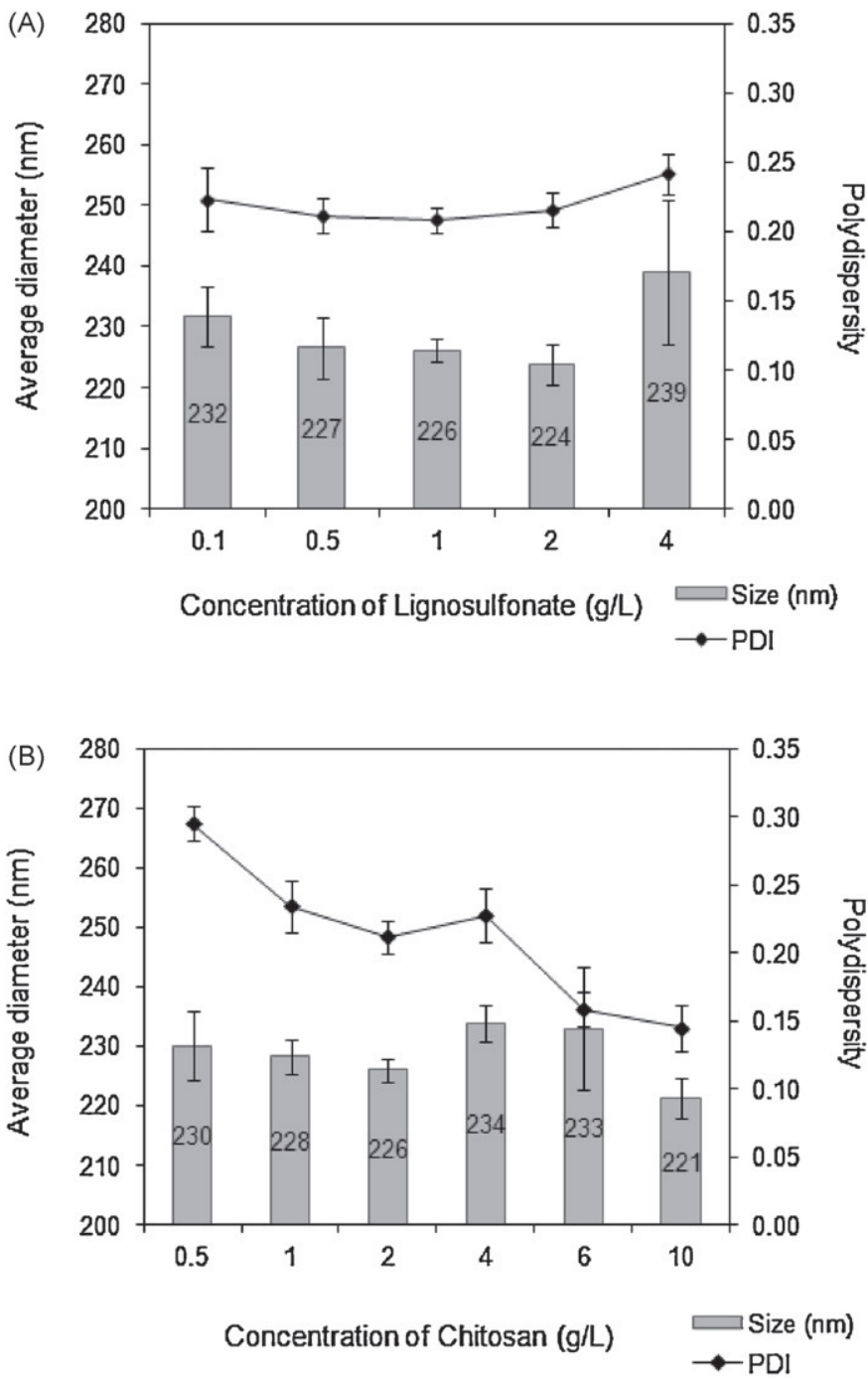

Fig. 4. Mean particles diameter and polydispersity of CS-LS microspheres as a function of concentration of chitosan (A) and lignosulfonates (B).

determine the drug release behaviour from chitosan nanoparticles. Apart from this, the type and concentration of the crosslinking agent, the type of oil, additives (surfactant or emulsifier) and the molecular weight of chitosan are also important players in the controlled release of the drug [5].

In this work, to study the entrapment efficiency of the newly developed CS-LS nanoparticles, RNase A was used as a model protein. Various RNase A concentrations were tested (Table 1). The results indicate that the higher the concentration of initial RNase A solution the higher is the protein incorporation and loading capacity. Both size and polydispersity of particles increase with increasing RNase A entrapment into chitosan nanoparticles.

Table 1

Protein incorporation, loading capacity, average diameter and polydispersity of CS-LS nanoparticles loaded with different concentrations of RNase A.

\begin{tabular}{lllll}
\hline $\begin{array}{l}\text { Rnase A load } \\
\left(\mathrm{g} \mathrm{L}^{-1}\right)\end{array}$ & $\begin{array}{l}\text { Entrapped } \\
\text { protein }\left(\mathrm{g} \mathrm{L}^{-1}\right)\end{array}$ & $\begin{array}{l}\text { Loading } \\
\text { capacity }(\%)\end{array}$ & Size $(\mathrm{nm})$ & Polydispersity \\
\hline 0 & - & - & $236.9 \pm 6.5$ & $0.17 \pm 0.03$ \\
0.2 & 0.122 & 6.6 & $273.1 \pm 12.1$ & $0.22 \pm 0.07$ \\
0.5 & 0.222 & 11.9 & $304.1 \pm 8.0$ & $0.30 \pm 0.05$ \\
1.0 & 0.274 & 14.7 & $471.5 \pm 21.5$ & $0.62 \pm 0.06$ \\
2.0 & 0.800 & 43.0 & $481.1 \pm 43.2$ & $0.63 \pm 0.07$ \\
\hline
\end{tabular}
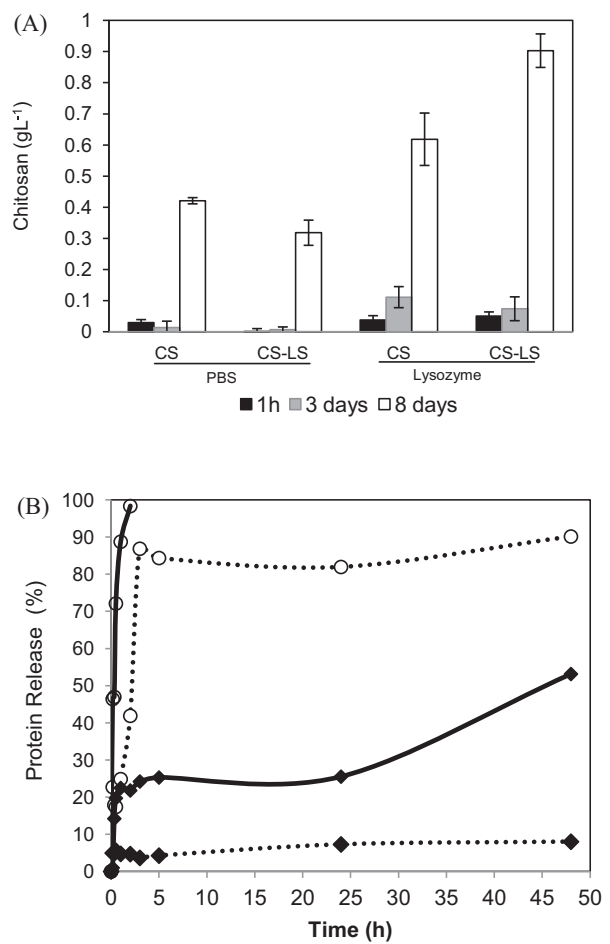

..0. CS-LS (0.2 gL-1 RNAse A) + PBS C CS-LS (0.2 gL-1 RNAse A) + Lysozyme

.*. CS-LS (2 gL-1 RNAse A) + PBS —CS-LS (2 gL-1 RNAse A) + Lysozyme

Fig. 5. (A) Stability of CS and CS-LS nanoparticles incubated in PBS or lysozyme for $1 \mathrm{~h}, 3$ days or 8 days. (B) Releasing profile of RNase A protein from the CS-LS nanoparticles in the presence of $0.5 \mathrm{~g} \mathrm{~L}^{-1}$ lysozyme (solid lines) or in the absence of lysozyme (just PBS buffer, dashed lines).

The mechanism of drug molecules entrapment in nanoparticles is an important aspect to be determined. The drugs either bind to the surface or are encapsulated inside the nanoparticles. The ultrasound-induced nanoparticles, however, may incorporate load in both ways. The ultrasound technique is known to generate aqueous suspensions of biopolymer microcapsules filled with water-insoluble liquids [26]. Therefore, if the drug is liposoluble it will be encapsulated inside the nanoparticle while if the drug is water soluble it will remain at the aqueous-lipid interface.

In Fig. 5B, the releasing profiles of CS-LS nanoparticles loaded with either the lowest $\left(0.2 \mathrm{~g} \mathrm{~L}^{-1}\right)$ or the highest concentration $\left(2 \mathrm{~g} \mathrm{~L}^{-1}\right)$ of RNase A are depicted. A burst release was observed in the initial $4 \mathrm{~h}$ of incubation after which the protein release stabilised under all the conditions tested (Fig. 5B). The amount of RNase released from the particle loaded with $0.2 \mathrm{~g} \mathrm{~L}^{-1}$ RNase A was superior, approximately $100 \%$ when exposed to both lysozyme and PBS in comparison to the amount released from the particle loaded with the higher concentration of protein (around 50\% in the presence of lysozyme and $5 \%$ in PBS). These profiles may indicate that the entrapment mechanism is dependent on the drug concentration. At lower protein concentrations the entrapment at the surface of nanoparticles may be favoured, while at higher concentrations both surface and bulk protein entrapment may occur, explaining both the polydispersity of particles (Table 1 ) and the release behaviour observed with both concentrations (Fig. 5B). An alternative explanation can be the difference in surface area available for the lysozyme action. The surface area is higher for the lowest protein loads (the average diameter is lower), therefore more surface is available to the action of this enzyme.

Despite the fact that the fast release of a drug in a burst stage can be pharmacologically dangerous and economically inefficient [27], there are certain situations where the rapid release or high 


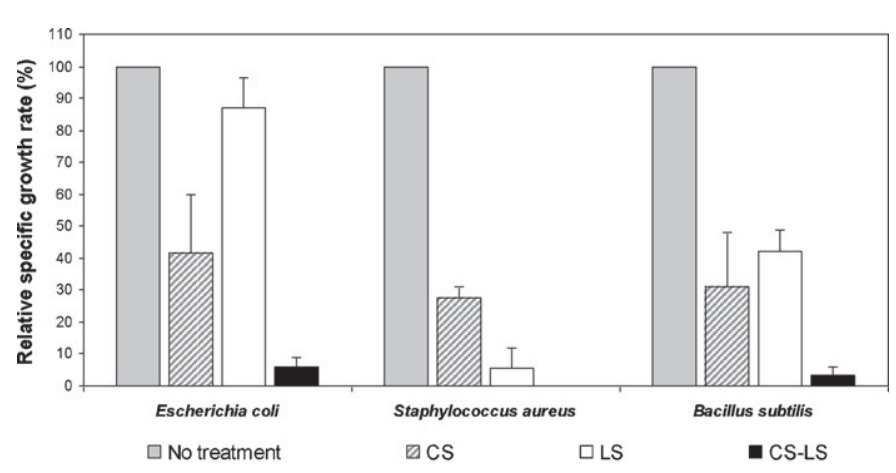

Fig. 6. Antimicrobial effect of CS, LS and CS-LS nanoparticles evaluated as the relative decrease in specific growth rate of each microorganism.

initial rates of delivery may be desirable. In cosmetic applications, the burst release is desired because it improves the drug penetration into the skin [28]. Other examples include wound management, encapsulation of flavours, triggered burst release and pulsatile release [27].

\subsection{Particles antimicrobial activity}

Fig. 6 shows the relative specific growth rates obtained for a Gram-negative bacteria E. coli, and for two Gram-positive bacteria $S$. aureus and $B$. subtilis when in contact with these particles. The specific growth rate obtained for each microorganism grown in MH medium containing the nanoparticles suspensions, was compared to the specific growth rates when the microorganism was simply grown in $\mathrm{MH}$ medium (no treatment). When microorganisms were grown in $\mathrm{MH}$ medium containing $100 \mu \mathrm{g} \mathrm{mL}^{-1}$ ampicilin, the culture absorbance did not change over time (data not shown). The growth rates of all tested microorganisms were decreased by exposure to all three types of nanoparticles. The concentration of CS, LS CS-LS in MH medium was $5 \times 10^{10}, 5 \times 10^{10}$ and $3 \times 10^{10}$ particles $/ \mathrm{mL}$, respectively, as determined by Nanoparticle Tracking Analysis.

Chitosan has long been known for its antimicrobial activity, although only recently nanoscaled chitosan and its derivatives have been characterised as antimicrobial agents against bacteria, viruses and fungi [29-32]. In relation to bacteria, the antimicrobial activity of chitosan has been reported to be higher against Gram-positive compared to Gram-negative bacteria [31]. Several antimicrobial mechanisms have been proposed for chitosan [29,31]. One mechanism involves positively charged chitosan particles interacting with negatively charged cell membranes, causing an increase in membrane permeability and eventually rupture and leakage of intracellular components [29]. This mechanism could explain the antimicrobial effect of CS and CS-LS nanoparticles, since they both have a positive zeta potential.

Comparing the growth rates between the Gram-negative and the Gram-positive bacteria, it appears that Gram-positives are also more susceptible to LS nanoparticles (Fig. 6). To our knowledge, this is the first report demonstrating the bacteriostatic activity of lignosulfonates. Whether the composition of the cell wall or other factors specific to these bacteria could be associated with the activity of this compound is not yet known. Since the charge of LS particles is negative, the mechanism must be different from the one that could explain the action of CS-containing nanoparticles.

Another interesting result was the fact that lignosulfonates and chitosan combined together in a particle have an enhanced antimicrobial effect against $E$. coli and B. subtilis. It is reasonable to suspect that the enhanced antimicrobial effect is due to the particular properties of CS-LS particles: high positive charge (around $+31 \mathrm{mV}$ ) and high surface area (average diameter $226 \mathrm{~nm}$ ).

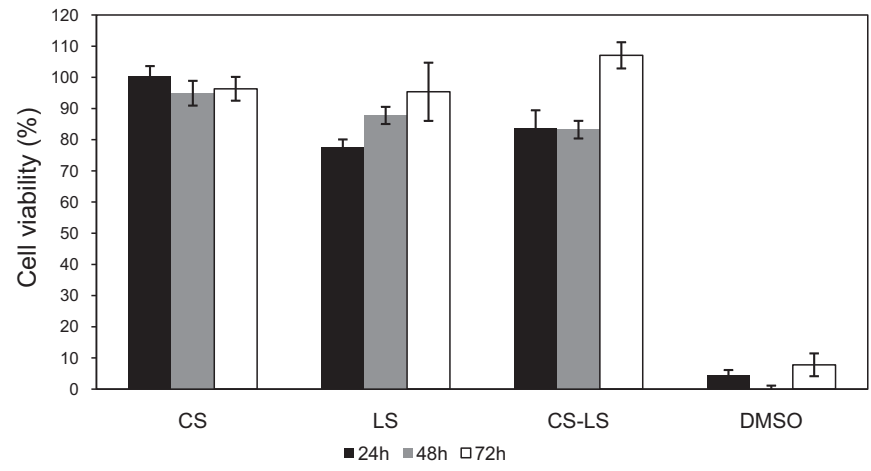

Fig. 7. Viability of human normal skin fibroblast cell line, BJ-5ta, after contact with sterilised suspensions of CS, LS and CS-LS nanoparticles during 24,48 and $72 \mathrm{~h}$. The viability is obtained by comparison with the negative control (no treatment).

\subsection{Particles cytotoxicity}

The biocompatibility of chitosan, lignosulfonates and chitosan-lignosulfonates particles was assessed on a human normal skin fibroblast cell line using AlamarBlue ${ }^{\circledR}$ assay, a simple and rapid method used to estimate the cytotoxicity of chemicals on cultured cells. Resazurin is reduced by cellular reductases to a pink-coloured chromophore, which is a direct measure of cell viability [33]. The nanoparticles suspensions of CS, LS and CS-LS, were found to be non-toxic after $72 \mathrm{~h}$ of exposure (Fig. 7). A slight toxicity was observed when cells where incubated with particles containing lignosulfonates after $24 \mathrm{~h}$ of incubation but were capable of recovery (Fig. 7). In fact, the recovery of fibroblasts from cytotoxic effects has been reported for lipid-based microparticles [34]. This behaviour indicates that CS-LS carriers are eventually biocompatible and can be safely applied to human skin.

\section{Conclusions}

Sonochemically prepared CS-LS nanoparticles were developed in this study as a potential carrier for drug delivery systems for both cosmetic and biomedical applications. A full characterisation of these particles was performed and after an optimisation process we were able to determine the conditions to attain carriers smaller than $230 \mathrm{~nm}$ in diameter. The electrostatic interactions between CS and LS were found to be determinant to develop biostable CS-LS nanoparticles, when compared with CS nanoparticles alone, which were found to be non-toxic and biocompatible and, furthermore, with increased antimicrobial activity. Apart from this, the CS-LS nanoparticles were found to display controlled release properties, depending on the amount of the incorporated protein model.

\section{Acknowledgments}

This work was financed by FP6 European project BioRenew (contract no. NMP2-CT-2006-026456), and by FEDER through POFC-COMPETE and by national funds from FCT through the project PEst-C/BIA/UI4050/2011. FCT - Portuguese Foundation for Science and Technology awarded scholarships to M.M. Fernandes (SFRH/BD/38363/2007), T. Matamá (SFRH/BPD/47555/2008) and A. Loureiro (SFRH/BD/81479/2011).

\section{References}

[1] L. Ma, C. Liu, Colloids Surf. B 75 (2010) 448-453.

[2] V.J. Mohanraj, Y. Chen, Trop. J. Pharm. Res. 5 (2006) 561-573.

[3] W.H. De Jong, P.J. Porm, Int. J. Nanomed. 3 (2008) 133-149.

[4] N. Kalpana, K.S. Shailendra, N.M. Dina, Chem. Pharm. Bull. 58 (2010) 1423-1430.

[5] V.R. Sinha, A.K. Singla, S. Wadhawan, R. Kaushik, R. Kumria, K. Bansal, S. Dhawan, Int. J. Pharm. 274 (2004) 1-33. 
[6] R. Nair, B.H. Reddy, C.K.A. Kumar, K.J. Kumar, J. Pharm. Sci. Res. 1 (2009) 1-12. 7] A. Nasti, N. Zaki, P. de Leonardis, S. Ungphaiboon, P. Sansongsak, M. Rimoli, N. Tirelli, Pharm. Res. 26 (2009) 1918-1930.

[8] H.-Q. Mao, K. Roy, V.L. Troung-Le, K.A. Janes, K.Y. Lin, Y. Wang, J.T. August, K.W. Leong, J. Control. Release 70 (2001) 399-421.

[9] J.C. Vengal, M. Srikumar, Trends Biomater. Artif. Organs 18 (2005) 237-241.

[10] D. Ekeberg, K.S. Gretland, J. Gustafsson, S.M. Brăten, G.E. Fredheim, Anal. Chim. Acta 565 (2006) 121-128.

[11] J.H. Lora, W.G. Glasser, J. Polym. Environ. 10 (2002) 39-48.

[12] W. Boerjan, J. Ralph, M. Baucher, Annu. Rev. Plant Biol. 54 (2003) 519-546.

[13] H. Faustino, N. Gil, C. Baptista, A.P. Duarte, Molecules 15 (2010) 9308-9322.

[14] E.S.K. Tang, M. Huang, L.Y. Lim, Int. J. Pharm. 265 (2003) 103-114.

[15] K.S. Suslick, M.W. Grinstaff, J. Am. Chem. Soc. 112 (1990) 7807-7809.

[16] O.H. Lowry, N.J. Rosebrough, A.L. Farr, R.J. Randall, J. Biol. Chem. 193 (1951) 265-275.

[17] A. Grenha, B. Seijo, C. Serra, C. Remuñán-López, Biomacromolecules 8 (2007) 2072-2079.

[18] A. Nel, T. Xia, L. Mädler, N. Li, Science 311 (2006) 622-627.

[19] A. Magrez, S. Kasas, V. Salicio, N. Pasquier, J.W. Seo, M. Celio, S. Catsicas, B. Schwaller, L. Forró, Nano Lett. 6 (2006) 1121-1125.

[20] N. Tran, T.J. Webster, J. Mater. Chem. 20 (2010) 8760-8767.
[21] T.W. Prow, J.E. Grice, L.L. Lin, R. Faye, M. Butler, W. Becker, E.M.T. Wurm, C. Yoong, T.A. Robertson, H.P. Soyer, M.S. Roberts, Adv. Drug Deliv. Rev. 63 (2011) 470-491.

22] J. Hankiewicz, E. Swierczek, Clin. Chim. Acta 57 (1974) 205-209.

[23] M. Papini, S. Simonetti, S. Franceschini, L. Scaringi, M. Binazzi, Arch. Dermatol. Res. 272 (1981) 167-170.

[24] J.H. Hamman, Mar. Drugs 8 (2010) 1305-1322.

[25] N. Samoilova, M. Krayukhina, I. Yamskov, Interpolyelectrolyte complexes of chitosan, in: S.P. Davis (Ed.), Chitosan: Manufacture, Properties and Usage, Nova Science Publishers, Inc., New York, 2011.

[26] A. Gedanken, Chem. Eur. J. 14 (2008) 3840-3853.

[27] X. Huang, C.S. Brazel, J. Control. Release 73 (2001) 121-136.

[28] A. Gulbake, A. Jain, P. Khare, S.K. Jain, J. Microencapsulation 27 (2010) 226-233.

[29] L. Qi, Z. Xu, X. Jiang, C. Hu, X. Zou, Carbohydr. Res. 339 (2004) 2693-2700.

[30] W. Ye, J.H. Xin, P. Li, K.-L.D. Lee, T.-L. Kwong, J. Appl. Polym. Sci. 102 (2006) 1787-1793.

[31] M.E. Badawy, E.I. Rabea, T.M. Rogge, C.V. Stevens, G. Smagghe, W. Steurbaut, M Hofte, Biomacromolecules 5 (2004) 589-595.

[32] H.K. No, Y.P. Na, H.L. Shin, S.P. Meyers, Int. J. Food Microbiol. 74 (2002) 65-72.

[33] G.R. Nakayama, M.C. Caton, M.P. Nova, Z. Parandoosh, J. Immunol. Methods 204 (1997) 205-208.

[34] L.J. Solmesky, M. Shuman, M. Goldsmith, M. Weil, D. Peer, Nanotechnology 22 (2011) 494016 